\title{
AN UNSUPERVISED RETINAL VESSEL EXTRACTION AND SEGMENTATION METHOD BASED ON A TUBE MARKED POINT PROCESS MODEL
}

\author{
Tianyu Li, Mary Comer
}

\author{
School of Electrical and Computer Engineering \\ Purdue University, West Lafayette, IN, USA
}

\author{
Josiane Zerubia \\ Inria and Université Côte d'Azur \\ Sophia-Antipolis, 06902, Cedex, France
}

\begin{abstract}
Retinal vessel extraction and segmentation is essential for supporting diagnosis of eye-related diseases. In recent years, deep learning has been applied to vessel segmentation and achieved excellent performance. However, these supervised methods require accurate hand-labeled training data, which may not be available. In this paper, we propose an unsupervised segmentation method based on our previous connected tube marked point process (MPP) model. The vessel network is extracted by the connected-tube MPP model first. Then a new tube-based segmentation method is applied to the extracted tubes. We test this method on STARE and DRIVE databases and the results show that not only do we extract the retina vessel network accurately, but we also achieve high Gmeans score for vessel segmentation, without using labeled training data.
\end{abstract}

Index Terms - Retinal vessel segmentation, vessel network extraction, connected tube model, marked point process

\section{INTRODUCTION}

Vessel extraction and segmentation in retina fundus images are essential for aiding analysis of eye-related pathology, such as hypertension, arteriosclerosis and diabetic retinopathy [1]. Many segmentation methods have been proposed over the past decades. In general, these methods can be classified as supervised or unsupervised methods, based on whether training data is needed or not.

Unsupervised methods are mostly rule-based, taking advantage of the contrast information, vessel structure and other features. Hoover et al. [2] segment the vessels by applying a 2D filter to the retinal images. Yin et al. [3] apply a probabilistic tracking method for the segmentation. Neto et al.[4] propose a coarse-to-fine algorithm with morphological operations and curvature analysis.

Most supervised methods take vessel segmentation as a binary classification problem. Traditional machine learning methods, such as support vector machines (SVMs) [5] and the Radius-Based Clustering algorithm(RACAL) [6], have been applied to vessel segmentation, and achieve good performance. In recent years, with the development of deep learning and with the use of GPUs, convolutional neural networks (CNNs) have been widely applied to image segmentation, including retinal vessel segmentation. Liskowski, Oliveira et al. [7] segment the vessels by a fully convolutional neural network with a stationary wavelet transform. Li et al. [8] realize the segmentation by a U-Net [9] combined with residual blocks. The results from deep neural networks are promising. However, the performance depends largely on accurate hand-labeled data, which may not always be available. For example, when equipment is upgraded or an image is captured with a different acquisition method, the data collected could be very different. Also the deep neural network tends to learn the behavior of the expert who labels the data, but for different experts, the labeling could be different. Thus, it is still meaningful to do research on unsupervised methods, even though the results may not be as competitive as deep learning methods with training data.

In this paper, we propose an unsupervised vessel segmentation method based on our previous connected-tube MPP model [10]. The framework of this method is presented in Fig 1. The image is pre-processed with morphological operations first. Then the vessel network is extracted by our connected-tube marked point process (MPP) model. Finally, a tube-based binary segmentation algorithm is applied to the detected tube objects.

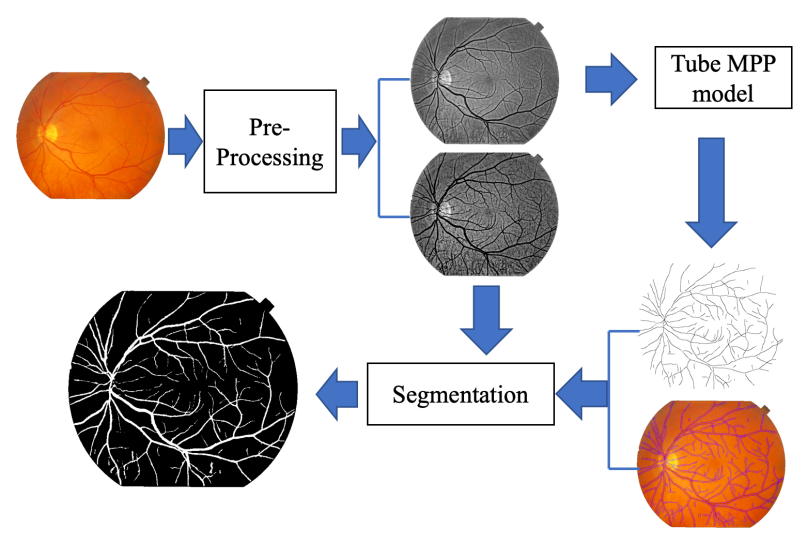

Fig. 1. The framework of the proposed method. 
The remainder of this paper is organized as follows: In Section 2, we present the pre-processing method and review the connected-tube MPP model. In Section 3, we introduce our tube-based segmentation algorithm. In Section 4, qualitative and quantitative results are given and discussed. Finally, conclusions are drawn in Section 5.

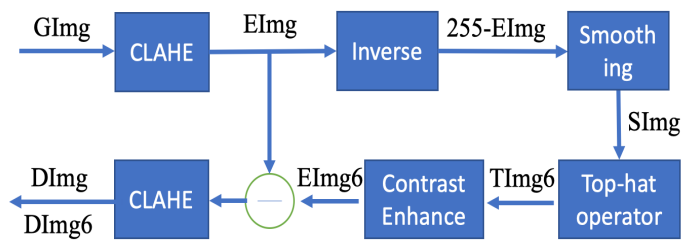

Fig. 2. The pipeline of preprocessing.

\section{VESSEL NETWORK EXTRACTION}

\subsection{Preprocessing}

Color retinal images usually exhibit some artifacts such as poor focus and camera misalignment [4]. Therefore, it is beneficial to enhance the image before processing. Since the green channel of the image provides the best contrast between the background tissue and vessel pixels [11], we process the image in this channel. We use a similar preprocessing method as in [4]. The main operations include: contrast limited adaptive histogram equalization (CLAHE) [12], Gaussian smoothing, and a top-hat operator. The preprocessing pipeline is given in Fig 2 . We enhance the contrast of the green channel image GImg by CLAHE first to get EImg. Then a Gaussian filter with size of $3 \times 3$ is applied to the inverted EImg (that is $255-E I m g$ ) to get SImg. A morphological top-operator with isotropic disk of radius 6 is applied to $S \operatorname{Img}$ to generate TImg6. We enhance TImg 6 by using a gray-level intensity mapping such that $1 \%$ of the data is saturated at the high and low intensity values to get $E \operatorname{Img} 6$. Finally we generate $2 \mathrm{im}$ ages as DImg $=C L A H E(E I m g-0.22 E I m g 6)$ and $D \operatorname{Img} 6=C L A H E(E \operatorname{Img}-E \operatorname{Img} 6)$. DImg is fed to the connected-tube MPP model for vessel network extraction. DImg6 is used for segmentation of vessels, as the pixels of a vessel tend to be darker than the background. One example (the im0163 image in STARE) of the preprocessing result can be seen in Fig 3: (a), (b) and (c).

\subsection{Connected tube-MPP model}

The connected-tube MPP model can be used for curvilinear structure extraction in both 2D and 3D images. In this section we briefly review the connected-tube MPP model and show its performance on retinal vessel extraction. For the 2D image, let $Y$ denote the observed image, $S$ the image

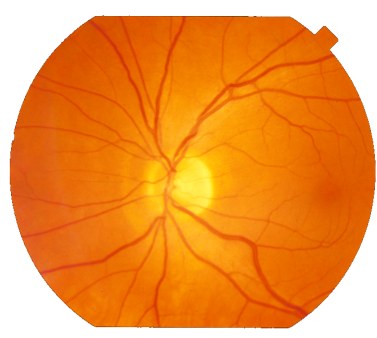

(a) Color image.

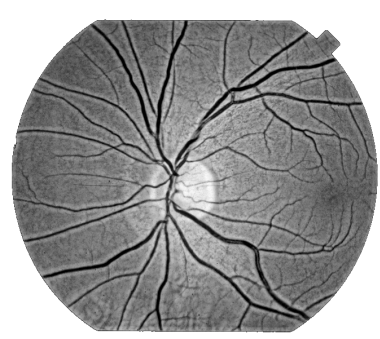

(c) DImg.

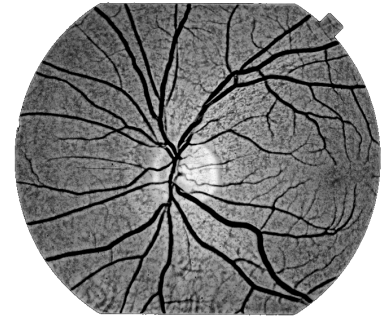

(b) DImg6.

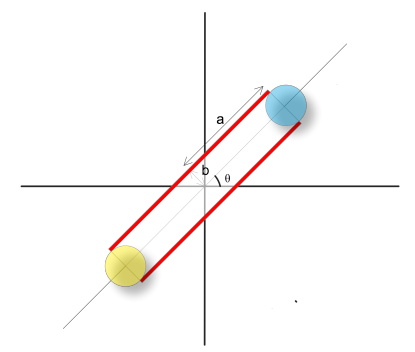

(d) Shape model of a tube.

Fig. 3. Example of image preprocessing for im0163 in STARE and the shape tube model.

lattice, $S \subset R^{2}$. A point process on $S$ is a set of points $\left\{S_{1}, S_{2}, \ldots, S_{n}\right\}$ in $S$, with the random variable $S_{i}$ representing the random location of the $i$ th point. In a connectedtube marked point process, each point $S_{i}$ is associated with a tube mark, which consists of random variables $(a, b, \theta)$ from a mark space $M=\left[a_{\min }, a_{\max }\right] \times\left[b_{\min }, b_{\max }\right] \times[0, \pi]$ describing a tube object located at $S_{i}$. As in Fig 3 (d), $a, b$ are the major and minor axes, which control the shape of the tube, and $\theta$ controls the orientation. The white region in Fig 3 (d) is the inner area, the red region is the outer area, which has width of 2 pixels, the blue region and yellow region are the front connection area and back connection area, respectively. A tube object is defined as a vector $w_{i}=\left(S_{i}, M_{i}\right) \in W$, where $W \subseteq S \times M$. Let $\Omega_{W}$ be the configuration space, which denotes the space of all possible realizations in $W$. Then $w=\left(w_{1}, w_{2}, \ldots, w_{n}\right) \in \Omega_{W}$ is a possible object configuration, where $n$ is the number of objects in this configuration. The density of the marked point process is given by

$$
f(w \mid y)=\frac{1}{Z} \exp \left\{-V_{d}(y \mid w)-V_{p}(w)\right\}
$$

where $Z$ is the normalizing constant, $V_{p}(w)$ is the prior energy introducing the prior knowledge on the object configuration. $V_{d}(y \mid w)$ is the data energy, which describes how well the objects fit the observed image. As in [10] we include the overlap prior, tube length prior and connection prior. The optimization of the model is realized by the Birth and Death kernel and Perturbation kernel. More detail about the model is stated in [10]. 
We input the preprocessed image DImg into the connectedtube model to extract the vessel networks. Fig 4 presents the extracted vessels from STARE-im0163. By drawing all the tubes (including the inner and outer area) as a binary image, we get 0.8819 recall and 0.8035 accuracy on average for the STARE dataset. Since the tube model does not segment the image pixel-wise, the accuracy is low at this object level. Though the accuracy is not high, it gives us the position of most vessels and their approximate width, which provide useful information and are helpful for fine segmentation of vessels.

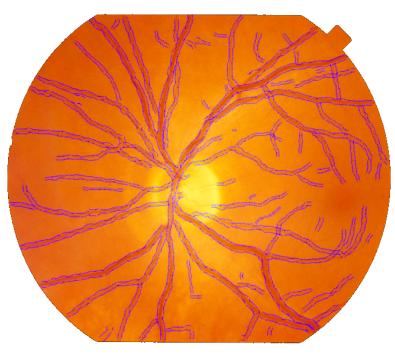

(a) Detected tube network.

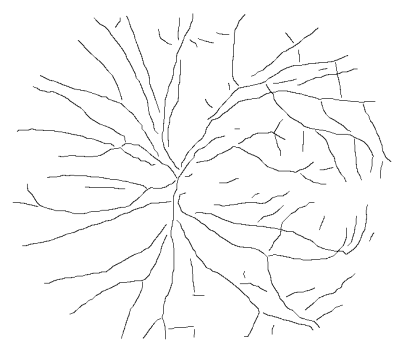

(b) Skeleton of vessels.
Fig. 4. Example of vessel extraction.

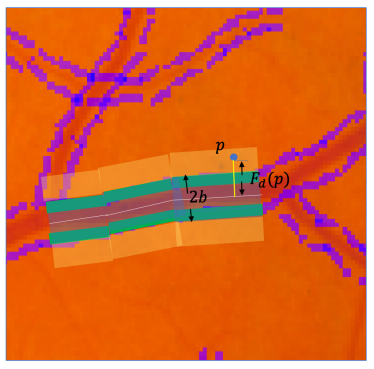

Fig. 5. Illustration of a segmentation area.

\section{VESSEL SEGMENTATION}

With the vessel network extracted using the connected-tube MPP model, the tubes with bad connection or bad contrast are removed and then we segment the vessels from the expanded tube object area. To ensure the vessels are contained in the object area, we expand the width of the detected tubes by 4 pixels. And each step, we take three connected tubes for segmentation, as shown in Fig 5. The three green tubes are connected, and the segmentation area includes both the tube object area (inner shaded area) and the expanded area (orange shaded area).

We deal with the segmentation task as a clustering problem. Given the pixels in the segmentation area, we partition these pixels into two clusters, cluster $C_{1}$ for vessel pixels (labeled as 1) and cluster $C_{0}$ for background pixels (labeled as 0 ). The clustering process is realized by a K-means [13] framework with a distance measure defined hereafter.

Due to the distortions in the vessel images, such as central vessel reflex and noisy vessel boundaries, it is ineffective to use gray values $F_{g}$ as the only feature for clustering. To deal with the distortions, we introduce two other features to describe each observation. One is location-based feature $F_{d}$, which is defined as the Euclidean distance between the pixel and the nearest center line of the tube objects. The other one $F_{r}$ is the ratio of pixels belonging to $C_{1}$ among a pixel's 8 neighbor pixels. $F_{d}$ encourages the pixels that are far away from the center line to be labeled as $0 . F_{r}$ guarantees the consistency between close pixels.

With $F_{g}, F_{d}, F_{r}$, we define the distance between a pixel $p$ and cluster $C_{0}$ by Eq. (2) and the distance between $p$ and $C_{1}$ using Eq. (3).

$$
\begin{aligned}
& D_{0}(p)=w_{g} \operatorname{Dist}_{0}(p)+w_{d}(1-\operatorname{Rad}(p))+w_{r} F_{r}(p) \\
& D_{1}(p)=w_{g} \operatorname{Dist}_{1}(p)+w_{d} \operatorname{Rad}(p)+w_{r}\left(1-F_{r}(p)\right)
\end{aligned}
$$

where $w_{g}, w_{d}, w_{r}$ are weights, $\operatorname{Dist}_{0}(p)$ and $\operatorname{Dist}_{1}(p)$ measure the gray distance of $p$ to the two clusters:

$$
\begin{aligned}
& \operatorname{Dist}_{0}(p)=1-\exp \left(\frac{-\left(F_{g}(p)-u_{0}\right)^{2}}{w_{g} \delta_{0}^{2}+1}\right) \\
& \operatorname{Dist}_{1}(p)=1-\exp \left(\frac{-\left(F_{g}(p)-u_{1}\right)^{2}}{w_{g} \delta_{1}^{2}+1}\right)
\end{aligned}
$$

$\operatorname{Rad}(p)$, defined in Eq. 6, measures the location distance between $p$ and cluster $C_{1}$. The location distance between $p$ and cluster $C_{0}$ is given as $1-\operatorname{Rad}(p)$.

$$
\operatorname{Rad}(p)=1-\exp \left(\frac{-F_{d}(p)^{2}}{w_{c} R(p)^{2}-0.5}\right)
$$

where $w_{g}$ and $w_{c}$ are weights; $R(p)$ is the half width $b$ of the tube that is close to $p ; u_{1}$ is the mean gray value of the pixels labeled as 1 , which we take as the center of $C_{1} ; u_{2}$ is the mean value of the pixels labeled as 0 , which is the center of $C_{0}$.

To initialize $u_{1}$ and $u_{0}$, we label pixels in the expanded area as 0 , and the pixels on the center line of tubes as 1 . Others pixels are considered to be unknown. The center line of tubes can be taken as the vessel skeleton, as in Fig 4 (b), for example. We update the label of each pixel in each iteration according to its distance to $C_{1}$ and $C_{0}$ respectively. If $\operatorname{Dist}_{1}(p)<\operatorname{Dist}_{0}(p)$, then pixel $p$ is labeled as 1 , otherwise it is labeled as 0 . Also $u_{1}, u_{0}, C_{1}$ and $C_{0}$ are updated at each iteration. The clustering process stops when maximum iteration 20 is reached or both $u_{1}$ and $u_{0}$ are not changed between previous iteration and current iteration. 


\section{EXPERIMENTS}

To demonstrate the performance of our method, we test it on two publicly available databases: STARE [2] and DRIVE [14]. STARE database contains 20 color retinal images (700 $\times 605)$ in which 10 of them are from individuals with medical conditions affecting the eye. The DRIVE database contains 40 color retinal images $(565 \times 584)$. The DRIVE images are divided into training and test sets, each including 20 images. For each database, there are two manual segmentations made by different experts. To compare our method with others' methods, we take the first observer's segmentation as ground truth; only pixels inside the field of view (FOV) are used. The sensitivity $\left(S e=\frac{T P}{T P+F N}\right), \operatorname{specificity}\left(S p=\frac{T N}{T N+F P}\right)$, $\operatorname{accuracy}\left(A c c=\frac{T P+T N}{N}\right)$, and G-mean $(G=\sqrt{S e \times S p})$, are used for performance evaluation and comparison. $\mathrm{N}$ is the total pixels inside the FOV, TP is true positive, TN is true negative, $\mathrm{FN}$ is false negative and FP is false positive.

The parameters for the connected-tube MPP model are set as $a_{\min }=3, a_{\max }=16, b_{\min }=1, b_{\max }=6$. For the segmentation algorithm, we set $w_{g}=0.58, w_{d}=0.27, w_{r}=$ $0.15, w_{g}=1.75, w_{c}=1.63$ by trial and error.

Fig 6 shows the qualitative results for one retinal image im0077 from STARE. We can see that the vessel networks are extracted accurately by our connected-tube MPP model. For this image, the segmentation scores are $S e=0.9220, S p=$ $0.9549, A c c=0.9514, G=0.9383$.

The comparison of our method with state-of-the-art algorithms (supervised methods: Fraz et al.[15], Orlando et al. [16], Oliveira et al. [7], Li et al. [8], and unsupervised methods: Zhang et al. [17], Neto et al. [4]) on STARE and DRIVE datasets are given in Table 1 and Table 2. It can be seen that we get the best Se among all these methods and the best $\mathrm{G}$ score among the unsupervised methods. From our analysis, there are mainly two reasons for the relatively lower $\mathrm{Sp}$ and Acc scores. One is that the edge pixels tend to be classified as vessel pixels due to the top-hat operation in preprocessing; the other one is the noise caused by bad tissues. We will address these problems in future work.

Table 1. Results on STARE dataset (SV is supervised, USV is unsupervised)

\begin{tabular}{|c|c|c|c|c|c|}
\hline \multicolumn{2}{|r|}{ Method } & $\mathrm{Se}$ & $\mathrm{Sp}$ & Acc & G \\
\hline \multicolumn{2}{|r|}{ 2nd observer } & 0.8956 & 0.9381 & 0.9346 & 0.9166 \\
\hline$S$ & Fraz et al.(2012) & 0.7548 & 0.9763 & 0.9534 & 0.8584 \\
\hline \multirow[t]{3}{*}{ V } & Orlando et al.(2017) & 0.7680 & 0.9738 & 0.9515 & 0.8648 \\
\hline & Oliveira et al.(2018) & 0.8315 & 0.9858 & 0.9694 & 0.9053 \\
\hline & Li et al.(2019) & 0.8101 & 0.9795 & - & 0.8905 \\
\hline $\mathrm{U}$ & Zhang et al.(2016) & 0.7791 & 0.9758 & 0.9554 & 0.8720 \\
\hline$S$ & Neto et al.(2017) & 0.8344 & 0.9443 & - & 0.8876 \\
\hline V & Proposed method & 0.8394 & 0.9536 & 0.9422 & 0.8932 \\
\hline
\end{tabular}

Table 2. Results on DRIVE dataset (SV is supervised, USV is unsupervised)

\begin{tabular}{|c|c|c|c|c|c|}
\hline & Method & $\mathrm{Se}$ & $\mathrm{Sp}$ & Acc & G \\
\hline & 2nd observer & 0.7760 & 0.9725 & 0.9473 & 0.8687 \\
\hline $\bar{S}$ & Fraz et al.(2012) & 0.7406 & 0.9807 & 0.9480 & $\overline{0.8522}$ \\
\hline V & Orlando et al.(2017) & 0.7897 & 0.9684 & 0.9454 & 0.8745 \\
\hline & Oliveira et al.(2018) & 0.8039 & 0.9804 & 0.9576 & 0.8878 \\
\hline & Li et al.(2019) & 0.7969 & 0.9799 & - & 0.8837 \\
\hline $\mathrm{U}$ & Zhang et al.(2016) & 0.7743 & 0.9725 & 0.9476 & 0.8678 \\
\hline$S$ & Neto et al.(2017) & 0.7806 & 0.9629 & - & 0.8670 \\
\hline V & Proposed method & 0.8063 & 0.9529 & 0.9339 & 0.8761 \\
\hline
\end{tabular}

\section{CONCLUSIONS}

In this paper, an unsupervised segmentation algorithm for retina vessel segmentation is proposed. By applying connectedtube MPP model to the enhanced retina image, the vessel network can be extracted accurately. Then a tube-based segmentation algorithm is applied to the expanded tube object area for vessel segmentation. Experiments on STARE and DRIVE databases show the performance of the proposed method is better than other unsupervised segmentation algorithms in $\mathrm{G}$ score. We get high sensitivity but relatively lower accuracy and specificity. In future work, we expect to improve accuracy and specificity by analyzing the structure of the extracted tube networks and the contrast quality for each tube.

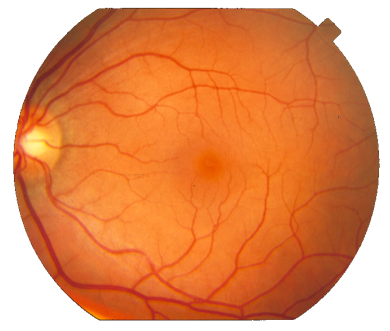

(a) Original image.

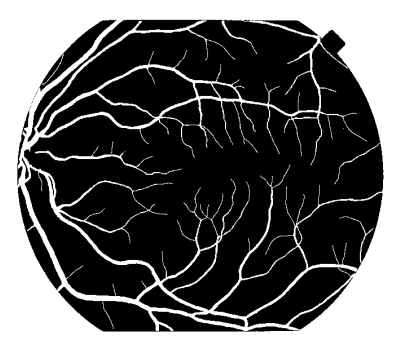

(c) Ground truth

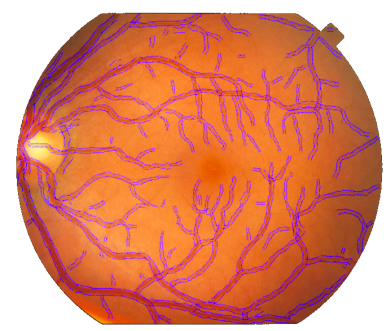

(b) Extracted tube network.

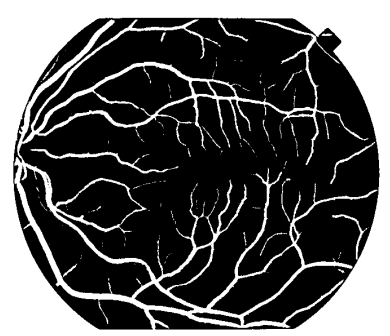

(d) Segmentation result.
Fig. 6. Qualitative result of im0077 in STARE. 


\section{REFERENCES}

[1] M. M. Fraz, P. Remagnino, A. Hoppe, B. Uyyanonvara, A.R. Rudnicka, C.G. Owen, and S.A. Barman, "Blood vessel segmentation methodologies in retinal images - a survey," Computer Methods and Programs in Biomedicine, vol. 108, no. 1, pp. 407 - 433, 2012.

[2] A. D. Hoover, V. Kouznetsova, and M. Goldbaum, "Locating blood vessels in retinal images by piecewise threshold probing of a matched filter response," IEEE Transactions on Medical Imaging, vol. 19, no. 3, pp. 203-210, March 2000.

[3] Y. Yin, M. Adel, M. Guillaume, and S. Bourennane, "A probabilistic based method for tracking vessels in retinal images," in Proceedings of the IEEE International Conference on Image Processing, 2010, pp. 4081-4084.

[4] L. C. Neto, G. L. B. Ramalho, J. F.S. R. Neto, R. M.S. Veras, and F. N.S. Medeiros, "An unsupervised coarseto-fine algorithm for blood vessel segmentation in fundus images," Expert Systems with Applications, vol. 78, pp. 182 - 192, 2017.

[5] E. Tuba, L. Mrkela, and M. Tuba, "Retinal blood vessel segmentation by support vector machine classification," in Proceedings of the IEEE International Conference Radioelektronika (RADIOELEKTRONIKA), April 2017, pp. 1-6.

[6] N. M. Salem, S. A. Salem, and A. K. Nandi, "Segmentation of retinal blood vessels based on analysis of the hessian matrix and clustering algorithm," in Proceedings of the 15th European Signal Processing Conference, Sep. 2007, pp. 428-432.

[7] A. Oliveira, S. Pereira, and C. A. Silva, "Retinal vessel segmentation based on fully convolutional neural networks," Expert Systems with Applications, vol. 112, pp. $229-242,2018$.

[8] D. Li, D. A. Dharmawan, B. P. Ng, and S. Rahardja, "Residual u-net for retinal vessel segmentation," in Proceedings of the IEEE International Conference on Image Processing, 2019, pp. 1425-1429.

[9] O. Ronneberger, P. Fischer, and T. Brox, "U-net: Convolutional networks for biomedical image segmentation," in Medical Image Computing and ComputerAssisted Intervention (MICCAI), Cham, 2015, pp. 234241 , Springer.

[10] T. Li, M. Comer, and J. Zerubia, "A Connected-Tube MPP Model for Object Detection with Application to Materials and Remotely-Sensed Images," in IEEE International Conference on Image Processing (ICIP). Oct. 2018, pp. 1323-1327, IEEE.
[11] J. V. B. Soares, J. J. G. Leandro, R. M. Cesar, H. F. Jelinek, and M. J. Cree, "Retinal vessel segmentation using the 2-d gabor wavelet and supervised classification," IEEE Transactions on Medical Imaging, vol. 25, no. 9, pp. 1214-1222, Sep. 2006.

[12] P. Vostatek, E. Claridge, H. Uusitalo, M. H. Kasari, P. Fält, and L. Lensu, "Performance comparison of publicly available retinal blood vessel segmentation methods," Computerized Medical Imaging and Graphics, vol. 55, pp. 2 - 12, 2017, Special Issue on Ophthalmic Medical Image Analysis.

[13] J. A. Hartigan and M. A. Wong, "Algorithm as 136: A k-means clustering algorithm," Journal of the Royal Statistical Society. Series C (Applied Statistics), vol. 28, no. 1, pp. 100-108, 1979.

[14] J. Staal, M. D. Abramoff, M. Niemeijer, M. A. Viergever, and B. van Ginneken, "Ridge-based vessel segmentation in color images of the retina," IEEE Transactions on Medical Imaging, vol. 23, no. 4, pp. 501-509, April 2004.

[15] M. M. Fraz, P. Remagnino, A. Hoppe, B. Uyyanonvara, A. R. Rudnicka, C. G. Owen, and S. A. Barman, "An ensemble classification-based approach applied to retinal blood vessel segmentation," IEEE Transactions on Biomedical Engineering, vol. 59, no. 9, pp. 2538-2548, Sep. 2012.

[16] J. I. Orlando, E. Prokofyeva, and M. B. Blaschko, "A discriminatively trained fully connected conditional random field model for blood vessel segmentation in fundus images," IEEE Transactions on Biomedical Engineering, vol. 64, no. 1, pp. 16-27, Jan 2017.

[17] J. Zhang, B. Dashtbozorg, E. Bekkers, J. P. W. Pluim, R. Duits, and B. M. ter Haar Romeny, "Robust retinal vessel segmentation via locally adaptive derivative frames in orientation scores," IEEE Transactions on Medical Imaging, vol. 35, no. 12, pp. 2631-2644, Dec 2016. 